\section{Efeito de intervenção no cumprimento das recomendações alimentares no primeiro ano de vida: ensaio clínico randomizado com mães adolescentes e avós maternas}

\author{
Effect of a healthy eating intervention on \\ compliance with dietary recommendations in the \\ first year of life: a randomized clinical trial with \\ adolescent mothers and maternal grandmothers
}

Efecto de intervención en el cumplimiento de las recomendaciones alimentarias durante el primer año de vida: ensayo clínico randomizado con madres adolescentes y abuelas maternas

\section{Resumo}

Avaliar o efeito de intervenção pró-alimentação saudável direcionada a mães adolescentes e avós maternas no cumprimento das recomendações alimentares no 1 o ano de vida. Ensaio clínico randomizado envolvendo 320 adolescentes, seus filhos e 169 avós maternas, quando em coabitação, randomicamente alocados para o grupo intervenção ou controle. A intervenção consistiu de seis sessões de aconselhamento em alimentação saudável durante o 1o ano de vida da criança, a primeira na maternidade e as demais aos 7, 15, 30, 60 e 120 dias, nos domicílios. As informações relativas à alimentação da criança foram obtidas mensalmente nos primeiros 6 meses e, depois, a cada 2 meses até a criança completar 12 meses, por meio de Questionário de Frequência Alimentar, além de perguntas sobre horários de refeições, consistência dos alimentos, cuidado com a higiene, preparo e conservação dos alimentos e conduta do cuidador com a alimentação da criança na vigência de doenças. Como desfecho, considerou-se o cumprimento dos Dez Passos para uma Alimentação Saudável: Guia Alimentar para Crianças Menores de Dois Anos, avaliado por meio de um escore. A média do somatório dos escores de cada passo obtidos no grupo intervenção foi maior que a do grupo controle; a intervenção dobrou a chance de o escore total ser maior ou igual à mediana. A coabitação com as avós não influenciou os escores, nem no grupo intervenção nem no grupo controle. A intervenção proposta teve efeito positivo no cumprimento dos Dez Passos, independentemente da participação da avó materna, mostrando que intervenções educativas podem melhorar a qualidade da alimentação das crianças no lo ano de vida.

Nutrição da Criança; Hábitos Alimentares; Ensaio Clínico
Leandro Meirelles Nunes 1,2

Álvaro Vigo 1

Luciana Dias de Oliveira 1

Elsa Regina Justo Giugliani 1

doi: 10.1590/0102-311X00205615

\author{
Correspondência \\ L. M. Nunes \\ Rua Cristóvão Colombo 603, apto. 201, Novo Hamburgo, RS \\ 93310-320, Brasil. \\ leandron@feevale.br \\ 1 Universidade Federal do Rio Grande do Sul, Porto Alegre, \\ Brasil. \\ 2 Universidade Feevale, Novo Hamburgo, Brasil.
}




\section{Introdução}

Tem-se observado rápida mudança nos padrões alimentares na infância, com piora da qualidade da dieta infantil de forma global, caracterizada por diminuição no consumo de frutas, legumes e fibras e aumento no consumo de alimentos processados ricos em açúcar, gordura saturada e sódio ${ }^{1,2}$. No Brasil, estimativas indicam que 47 a $65 \%$ das crianças consomem dieta de qualidade baixa ou intermediária para alimentos ou nutrientes 3,4. Essa situação pode estar implicada no aumento da prevalência de sobrepeso/obesidade e hipertensão arterial sistêmica, especialmente em crianças e adolescentes 5 . Essas duas condições, quando presentes na infância, tendem a persistir na vida adulta, aumentando a prevalência de doenças crônicas não transmissíveis e mortalidade precoce 6,7 .

Apesar das inúmeras evidências dos benefícios do aleitamento materno exclusivo (AME) nos primeiros 6 meses de vida, do aleitamento materno (AM) por 2 anos ou mais e da alimentação complementar saudável introduzida em tempo oportuno 8,9,10, os indicadores de AM e alimentação complementar no Brasil continuam aquém do desejável. O último inquérito nacional realizado nas capitais brasileiras e no Distrito Federal estimou duração mediana do AME em 52 dias, com menos de 10\% das crianças sendo amamentadas exclusivamente até os 6 meses de vida; e duração mediana do AM de 11,2 meses, com 45,5\% das crianças sendo amamentadas por 12 meses ou mais. O estudo detectou também início precoce da alimentação complementar, com 1/4 das crianças entre 3 e 6 meses já recebendo alimentos salgados e/ou frutas, $9 \%$ consumindo alimentos não saudáveis, como bolachas e salgadinhos, e aproximadamente metade recebendo outros leites entre 4 e 6 meses de vida 11 .

Diante desse cenário, o Ministério da Saúde do Brasil, com base em diretrizes da Organização Mundial da Saúde (OMS) 12, estabeleceu recomendações para a alimentação de crianças brasileiras menores de 2 anos, as quais são sumarizadas nos Dez Passos para uma Alimentação Saudável: Guia Alimentar para Crianças Menores de Dois Anos 13. Os objetivos dessas recomendações são aumentar as prevalências de AM e AME e melhorar a qualidade da alimentação complementar, além de contribuir para a formação de hábitos alimentares saudáveis 13 .

Pesquisas conduzidas em diferentes populações 14,15,16,17,18,19,20, inclusive na do presente estudo 18,19 , sugerem que maternidade na adolescência e coabitação com a avó materna da criança são possíveis fatores de risco tanto para menor duração do AM, quanto para práticas alimentares inadequadas no 1o ano de vida. Tal fato se deve, provavelmente, à dependência econômica das adolescentes em relação à sua família, o que muitas vezes implica, além da coabitação, a participação direta das avós na decisão, seleção e preparo dos alimentos 20; por isso, este estudo teve como objetivo testar o efeito de uma intervenção pró-AM e pró-alimentação complementar saudável, direcionada a mães adolescentes e avós maternas das crianças quando em coabitação, sobre a qualidade da alimentação das crianças no primeiro ano de vida, avaliada pelo cumprimento dos Dez Passos para uma Alimentação Saudável: Guia Alimentar para Crianças Menores de Dois Anos.

\section{Método}

Foi planejado um ensaio clínico randomizado envolvendo mães adolescentes, seus filhos e também as avós maternas das crianças quando em coabitação. As adolescentes foram recrutadas no alojamento conjunto da maternidade do Hospital de Clínicas de Porto Alegre, no Sul do Brasil, obedecendo aos seguintes critérios de inclusão: residir no Município de Porto Alegre, ter 19 anos de idade ou menos, gestação com feto único e amamentação iniciada durante a internação. Não foram incluídas no estudo as mães e recém-nascidos que foram separados na maternidade por problemas maternos ou neonatais, os bebês com peso de nascimento $<2.500 \mathrm{~g}$ e as mães que coabitavam com as avós paternas da criança, além de mães e crianças com problemas que impedissem ou dificultassem a amamentação, como, por exemplo, mães positivas para o HIV e crianças com más-formações congênitas.

Diariamente, entre maio de 2006 e janeiro de 2008, mães adolescentes que haviam dado à luz a seus bebês nas últimas 24 horas foram convidadas a participar do estudo. Após a obtenção de consentimento e entrevista inicial, a adolescente era classificada como pertencente a um dos dois seguintes grupos iniciais: coabitando com a avó da criança e não coabitando com a avó da criança. Posteriormente, foi realizado sorteio para sua alocação em grupo controle ou intervenção, em blocos de dois, 
ou seja, se uma mãe era sorteada para o grupo intervenção, a próxima mãe elegível necessariamente era incluída no grupo controle. Para o sorteio, utilizaram-se duas esferas de mesma textura e dimensões com os dizeres "sim" e "não", que eram retiradas de um invólucro de coloração escura pelo mesmo pesquisador que fazia a seleção inicial, impedindo a distinção visual ou tátil das mesmas. Dessa forma, as adolescentes eram distribuídas em quatro grupos distintos: (1) não coabitando com a avó materna, sem intervenção; (2) não coabitando com a avó materna, com intervenção direcionada apenas à adolescente; (3) coabitando com a avó materna, sem intervenção; (4) coabitando com a avó materna, com intervenção direcionada à adolescente e à avó. Estipulou-se que metade da amostra seria formada por adolescentes que coabitavam com as avós maternas das crianças e metade sem essa coabitação. A inclusão de grupos sem a presença da avó teve como finalidade avaliar o quanto do impacto da intervenção poderia ser atribuído apenas às adolescentes.

A intervenção consistiu em seis sessões de aconselhamento em AM e alimentação complementar saudável, com base nos Dez Passos 13. Cada sessão durava aproximadamente 30-40 minutos. A primeira ocorria na maternidade, as demais nos domicílios, aos 7, 15, 30, 60 e 120 dias de vida da criança, conduzidas sempre pelo mesmo profissional, tomando-se o cuidado de distribuir igualmente o número de sessões entre os profissionais. Na maternidade, as sessões aconteciam em momentos distintos para as mães e para as avós; nos domicílios, mães e avós recebiam a intervenção simultaneamente. As intervenções foram conduzidas por um dos quatro profissionais envolvidos nessa tarefa (um pediatra, duas enfermeiras e uma nutricionista), todos com ampla experiência em AM e alimentação infantil. De maneira informal, por meio de diálogos, as sessões abordavam vários aspectos do AM, dandose ênfase à importância e manutenção do AME nos primeiros 6 meses, mas também abordando os seguintes itens: frequência e duração das mamadas, fatores que interferem na produção de leite, técnica de amamentação, prevenção de traumas mamilares e ingurgitamento mamário, consequências do uso de chupeta, mamadeira e introdução precoce de outros alimentos, choro e comunicação do bebê. Na sessão oferecida aos 4 meses, era dada ênfase à alimentação complementar, com início a partir dos 6 meses, tendo como base as recomendações contidas nos Dez Passos. Dois álbuns seriados (um sobre AM e outro sobre alimentação complementar) foram elaborados especialmente para a pesquisa e serviram de apoio nas intervenções. Além disso, cartilhas sobre AM foram distribuídas (uma para as mães adolescentes e outra para as avós) após a primeira sessão da intervenção, ainda na maternidade, e uma cartilha sobre alimentação complementar, com algumas receitas para exemplificar os grupos alimentares e o preparo dos alimentos, foi distribuída após a sessão realizada aos 4 meses. As mães/avós dos grupos controle e intervenção receberam o mesmo atendimento hospitalar oferecido rotineiramente a todas as mães, em consonância com as normas e rotinas estabelecidas quando da certificação do hospital na Iniciativa Hospital Amigo da Criança.

O conteúdo da intervenção, tanto para as mães quanto para as avós, foi elaborado após conversas com vinte puérperas adolescentes e avós maternas das crianças. Elas foram ouvidas quanto aos seus conhecimentos, práticas, crenças, mitos, fantasias, dificuldades e expectativas relacionadas à alimentação da criança pequena. Com isso, foram criados questionários iniciais (para as mães e para as avós) e uma estratégia de intervenção, os quais foram testados em estudo piloto com dez mães adolescentes e avós. Após o piloto, foram feitos ajustes e adaptações, até se chegar à versão final.

Na maternidade, mães e avós, quando em coabitação, foram entrevistadas visando à obtenção de informações relativas a características sociodemográficas, aspectos relacionados ao acompanhamento pré-natal, parto e experiência prévia com amamentação. Dados sobre a alimentação da criança foram coletados mensalmente nos primeiros 6 meses e, depois, aos 8, 10 e 12 meses. Para isso, as mães/ cuidadores eram solicitadas a responder, por telefone - ou, na impossibilidade de contato telefônico, no domicílio, o que ocorreu em torno de $10 \%$ das entrevistas -, a um questionário relativo à alimentação das crianças no momento da entrevista contendo perguntas sobre consumo de leite materno, água, chás, sucos, fórmulas lácteas infantis, outros leites e outros alimentos, além de Questionário de Frequência Alimentar (QFA) semanal e perguntas relativas aos seguintes aspectos da alimentação: horários e frequência das refeições, consistência dos alimentos ofertados, higiene no preparo, armazenamento e conservação dos alimentos, e conduta do cuidador com a alimentação da criança na vigência de doenças ou convalescença.

Os entrevistadores eram cegos em relação ao grupo ao qual as mães e avós pertenciam. Com o intuito de checar a qualidade das informações, a coordenadora de campo sorteava mensalmente $5 \%$ 
das mães e solicitava que respondessem novamente a algumas perguntas-chave do questionário, tais como consumo de leite materno e de outros alimentos, alguns itens do QFA e conduta alimentar em caso de adoecimento da criança. Essa segunda entrevista ocorria num intervalo de até 30 dias e as respostas eram relacionadas ao momento da primeira entrevista e não ao momento atual da checagem. O banco de dados foi duplamente digitado no programa Microsoft Excel (Microsoft Corp., Estados Unidos) e posteriormente submetido à validação. A análise estatística foi realizada com o programa IBM SPSS for Windows (IBM Corp., Armonk, Estados Unidos), versão 23.0.

O cálculo do tamanho amostral baseou-se em uma prevalência de AME no primeiro mês no grupo controle de $56 \% 21$ e na estimativa de uma diferença de $25 \%$ na frequência dessa prática entre os grupos após a intervenção. Outros parâmetros utilizados no cálculo foram poder de $80 \%$ e nível de $95 \%$ de confiança, determinando um tamanho amostral de 48 duplas mãe-bebê em cada um dos quatro grupos. Para compensar eventuais perdas e possibilitar a realização de análises multivariadas, determinou-se um tamanho amostral de 72 duplas participantes para cada um dos quatro grupos.

O cumprimento dos Dez Passos foi utilizado como proxy da qualidade da alimentação complementar. Para avaliar o cumprimento do passo 1, utilizaram-se as respostas de todos os questionários de seguimento até o 6 o mês; com relação ao passo 2, utilizaram-se os nove questionários de seguimento; e para o restante dos passos (3 a 10), foram levadas em consideração as respostas dos questionários respondidos ao final do 6ọ, 8o, 10o e 12o mês de vida da criança. Para cada um dos Dez Passos, atribuiuse um escore de acordo com o cumprimento do passo. Os passos cumpridos integralmente em todos os questionários analisados receberam escore final de 2 pontos, os parcialmente cumpridos em pelo menos um dos questionários receberam escore final de 1 ponto, e os não cumpridos em pelo menos um dos questionários analisados não pontuaram (0 pontos) (Tabela 1). Dessa forma, o escore final de cada criança poderia variar de 0 a 20 pontos. As médias dos somatórios dos escores de cada passo obtidas nos grupos intervenção e controle foram comparadas mediante uso do teste t de Student; as frequências do cumprimento de cada um dos passos foram comparadas com utilização do teste $t$ de Student com cálculo dos resíduos ajustados. Adotou-se o nível de $5 \%$ de significância $(\mathrm{p} \leq 0,05)$.

Para a análise dos dados, considerou-se a intenção de tratar. Em um primeiro momento, para testar se a randomização foi afetada pelas perdas ao longo do seguimento, foram comparadas as características dos indivíduos dos grupos controle e intervenção que permaneceram no estudo até o final. Essa comparação foi realizada utilizando-se o teste do qui-quadrado com correção de Yates.

Para estimar o efeito da intervenção sobre o cumprimento dos Dez Passos, foi utilizado o modelo de regressão multivariada de Poisson com estimação robusta, adotando-se como ponto de corte a mediana do escore de toda a população estudada. Como se observou que a participação da avó materna não influenciava os resultados, optou-se por considerar apenas dois grupos, controle e intervenção, independentemente da coabitação com as avós maternas, com o intuito de dar mais robustez às análises; e incluir no modelo multivariável a coabitação com a avó, haja vista não ter havido randomização dessa variável. Primeiramente, testou-se um modelo não ajustado (modelo 1); em seguida, foi incluído no modelo o escore de propensão (modelo 2); e, finalmente, ao modelo 2 foi adicionada a coabitação com a avó (modelo 3). O escore de propensão, muito utilizado em estudos observacionais para compensar a falta de aleatoriedade, pode ser extremamente útil para controlar confundimento em experimentos aleatórios, especialmente quando há muitos fatores de confusão e/ou em pequenos estudos 22. Como neste estudo a randomização foi realizada no início do e houve perdas, a inclusão do escore de propensão no modelo foi um recurso adicional para controlar o potencial de confusão em um eventual desequilíbrio entre os grupos 22,23. O escore de propensão foi estimado por meio de regressão logística, considerando os seguintes preditores: idade, cor da pele, nível educacional e parição da mãe; peso de nascimento e via de nascimento da criança; e coabitação com o pai da criança.

A pesquisa, registrada no ClinicalTrials.gov sob o número NCT00910377, foi aprovada pela Comissão de Pesquisa e Ética em Saúde do Hospital de Clínicas de Porto Alegre (projeto 02-389). 


\section{Tabela 1}

Dez Passos para uma Alimentação Saudável: Guia Alimentar para Crianças Menores de Dois Anos e critérios utilizados na pontuação de cada passo baseados nas respostas dos seguintes questionários de seguimento: passo 1 (primeiros 6 meses), passo 2 (todos os questionários), passo 3 a 10 (6으, 8으, 10o e 12 o mês).

\section{Passos}

\section{Pontuação}

1. Dar somente leite materno até os 6 meses, sem oferecer água, chás ou qualquer outro alimento.

2. A partir dos 6 meses, oferecer de forma lenta e gradual outros alimentos, mantendo o leite materno até os 2 anos de idade ou mais.

3. A partir dos 6 meses, dar alimentos complementares (cereais, tubérculos, carnes, frutas e legumes) três vezes ao dia se a criança receber leite materno e cinco vezes ao dia se estiver desmamada.

4. A alimentação complementar deve ser oferecida sem rigidez de horários, respeitando-se sempre a vontade da criança.
0 - Duração do aleitamento materno exclusivo inferior a 4 meses.

1 - Duração do aleitamento materno exclusivo entre 4 e 5 meses.

2 - Duração do aleitamento materno exclusivo de 6 meses.
0 - Introdução da alimentação complementar < 6 meses e duração da amamentação < 12 meses.

1 - Introdução da alimentação complementar $<6$ meses e duração do aleitamento materno $\geq 12$ meses ou introdução da alimentação complementar $\geq 6$ meses e duração da amamentação < 12 meses. 2 - Introdução da alimentação complementar $\geq 6$ meses e duração da amamentação $\geq 12$ meses.

0 - Criança recebe alimentos $\leq$ dois grupos dos cinco recomendados. 1 - Criança recebe alimentos de três ou quatro grupos dos cinco recomendados. 2 - Criança recebe alimentos dos cinco grupos recomendados.

0 - Alimentação em horários predeterminados, não respeitando a vontade da criança; criança é forçada a comer e, em caso de não aceitação, o alimento é oferecido apenas no próximo horário de refeição. 1 - Em caso de não aceitação do alimento no horário predeterminado, é oferecido outro alimento, incluindo alimentos lácteos.

2 - Flexibilidade de horário na alimentação; em caso de recusa, o mesmo alimento é oferecido um pouco mais tarde.

5. A alimentação complementar deve ser espessa desde o início e oferecida de colher; começar com consistência pastosa (papas/purês) e gradativamente aumentar a consistência até chegar à alimentação da família.

6. Oferecer à criança diferentes alimentos ao dia. Uma alimentação variada é uma alimentação colorida *

7. Estimular o consumo diário de frutas, verduras e legumes nas refeições.
0 - Alimentos liquidificados ou passados na peneira, em qualquer idade.

1 - Qualquer condição diferente das equivalentes às pontuações 0 e 2.

2 - Alimentos bem amassados entre 6 e 8 meses, levemente amassados ou picados entre 8 e 12 meses e na consistência dos alimentos da família aos 12 meses.

0 - Consumo de alimentos com uma ou duas cores.

1 - Consumo de alimentos com três ou quatro cores.

2 - Consumo de alimentos com cinco cores.

0 - Não consome frutas, verduras e legumes.

1 - Qualquer condição diferente das equivalentes às pontuações 0 e 2 .

2 - Consome frutas, verduras e legumes cinco vezes ou mais na semana.

8. Evitar açúcar, café, enlatados, frituras, 0 - Consome sal em quantidade não moderada, açúcar e alimentos não saudáveis ** mais que uma vez refrigerantes, balas, salgadinhos e outras guloseimas nos primeiros anos de vida. Usar sal com moderação. por semana.

1 - Qualquer condição diferente das equivalentes às pontuações 0 e 2

2 - Consome sal em quantidade moderada e consome açúcar e alimentos não saudáveis ** até no máximo uma vez por semana.

9. Cuidar da higiene no preparo e manuseio dos alimentos; garantir seu armazenamento e conservação adequados.

10. Estimular a criança doente e convalescente a se alimentar, oferecendo sua alimentação habitual e seus alimentos preferidos, respeitando sua aceitação.

0 - A pessoa que prepara o alimento não higieniza suas mãos nem as da criança, reaproveita o alimento presente no prato da criança após a refeição e não oferece água tratada.

1 - Qualquer condição diferente das equivalentes às pontuações 0 e 2 .

2 - A pessoa que prepara o alimento higieniza suas mãos e as da criança, não reaproveita o alimento presente no prato da criança após a refeição e oferece água tratada.

0 - Cuidador força a criança a comer quando está doente, não incentiva a alimentação ou faz pausa alimentar.

1 - Cuidador aumenta a frequência de mamadas ou líquidos, mas não incentiva a alimentação quando a criança está doente.

2 - Cuidador incentiva a alimentação, oferece os alimentos preferidos da criança com maior frequência inclusive os líquidos, quando ela está doente.

* Cores dos alimentos: branco, vermelho, amarelo, verde e roxo;

** Alimentos não saudáveis: refrigerante, bolachas recheadas, doces, salgadinhos, enlatados, embutidos, café e frituras. 


\section{Resultados}

A Figura 1 apresenta o fluxograma do processo de seleção de participantes para o ensaio clínico randomizado, desde o recrutamento das mães adolescentes até a avaliação feita quando as crianças completavam 12 meses de idade. Das 320 mães adolescentes que entraram no estudo, 234 permaneceram até a avaliação feita no 12 o mês (final do estudo). Houve equilíbrio nas perdas entre os grupos (intervenção: 42/160, 26,2\%; controles: 44/160, 27,5\%; p = 0,251).

As características dos participantes dos grupos controle e intervenção que concluíram o estudo foram similares. Não houve diferença estatisticamente significativa ( $p>0,05)$ entre os grupos quanto às características maternas (raça, idade, renda, escolaridade, número de consultas pré-natais e número de filhos), características das crianças (tipo de parto, sexo e peso ao nascer) e coabitação com a avó materna e com o companheiro. À exceção das características das crianças, que foram coletadas de prontuário hospitalar, e do número de consultas pré-natais, que foi obtido do registro na carteira de consultas obstétricas, as demais informações foram autodeclaradas.

As características dos participantes que permaneceram no estudo até o final foram como segue: média da idade materna, 17,5 \pm 1,4 anos; mãe de cor branca, 63,2\% ( $\mathrm{n}=151$ ); média da renda familiar, 2,5 salários mínimos; mãe com Ensino Fundamental completo (8 anos de escolaridade), 55,6\% ( $\mathrm{n}=$ 133); mãe primigesta, $84,5 \%(\mathrm{n}=202)$; parto vaginal, $77,8 \%(\mathrm{n}=186)$; criança do sexo masculino, $51,9 \%$ ( $\mathrm{n}=124)$; e coabitação com marido/companheiro, $60,7 \%(\mathrm{n}=145)$.

\section{Figura 1}

Fluxograma ilustrando o processo de seleção de participantes desde o recrutamento até a última avaliação da criança, aos 12 meses.

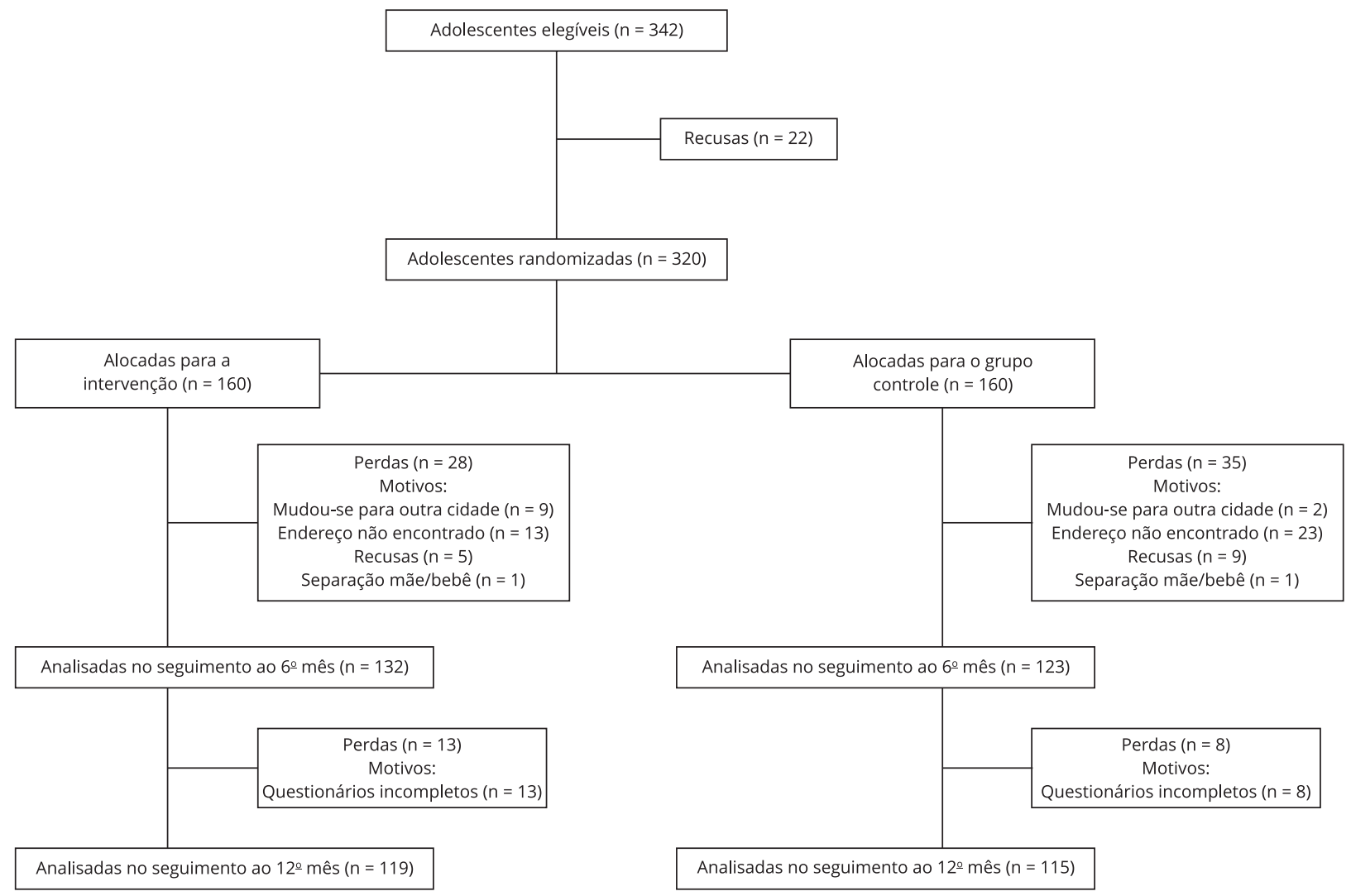


O escore relativo ao cumprimento dos passos variou de 6 a 19, com mediana de 11. Houve diferença significativa, de aproximadamente 2 pontos, entre os escores obtidos nos grupos intervenção $e$ controle $(p=0,00)$. A coabitação com as avós não influenciou os escores, nem no grupo intervenção nem no grupo controle (Tabela 2).

Os percentuais de cumprimento de cada um dos Dez Passos nos dois grupos são apresentados na Tabela 3. Ocorreu diferença estatisticamente significativa entre os grupos controle e intervenção nos passos 1 a 6 .

A Tabela 4 mostra os resultados da análise multivariada testando o efeito da intervenção sobre o cumprimento dos Dez Passos. Em média, a chance de um indivíduo do grupo intervenção possuir um escore maior ou igual à mediana (escore 11) foi cerca de 2 vezes maior em relação aos indivíduos do grupo controle. Observou-se, também, que a inclusão do escore de propensão e da presença da avó materna no modelo não afetaram o desfecho, demonstrando que o fator temporal mais importante para determinar um escore maior na população estudada foi a intervenção.

\section{Discussão}

Este estudo demonstrou que é possível melhorar a qualidade da alimentação complementar de filhos de mães adolescentes mediante o oferecimento de múltiplas sessões de aconselhamento em AM e alimentação complementar saudável nos primeiros 4 meses de vida das crianças. As crianças cujas mães receberam o aconselhamento pró-alimentação saudável obtiveram escores maiores no cumprimento dos Dez Passos, preconizados pelo Ministério da Saúde.

Este estudo corrobora os achados de outros dois ensaios clínicos: um realizado no Brasil, em população semelhante à deste estudo 24,25 , e outro realizado na Inglaterra 26 . A intervenção testada no estudo brasileiro, oferecida em dez visitas domiciliares ao longo do primeiro ano de vida da criança, aumentou o consumo de frutas e hortaliças e tornou a dieta mais diversificada aos 3-4 anos de idade. Em Londres, uma intervenção com encontros mensais dos 3 aos 12 meses teve efeito positivo no consumo de frutas e hortaliças aos 18 meses. No entanto, ambos os estudos, diferentemente do presente, incluíram mães de todas as idades e não envolveram as avós das crianças.

Tem-se conhecimento de apenas um ensaio clínico randomizado que testou uma intervenção de promoção da alimentação complementar saudável voltada às mães adolescentes. $\mathrm{O}$ estudo, realizado com 181 mães adolescentes, primíparas e de baixa renda, nos Estados Unidos, tinha como intervenção visitas domiciliares realizadas a cada 2 semanas durante 1 ano, por duas mulheres que tiveram seus filhos também durante a adolescência. Além de conversas, as mães assistiam a um videotape que abordava várias questões relativas à amamentação e introdução da alimentação complementar, bem como particularidades da mãe adolescente e de sua relação com os familiares. Uma cópia do vídeo era disponibilizada para que as adolescentes pudessem discutir os assuntos abordados com seus familiares em algum momento posterior. Na entrevista realizada aos 3 meses de idade da criança, mães que

\section{Tabela 2}

Escores (média + desvio-padrão) relativos ao cumprimento dos Dez Passos para uma Alimentação Saudável: Guia Alimentar para Crianças Menores de Dois Anos, analisados em pares, de acordo com o grupo e a condição de coabitação com a avó materna da criança. Porto Alegre, Rio Grande do Sul, Brasil, 2006/2009.

\begin{tabular}{lcccc}
\hline & Amostra total $(\mathbf{N}=\mathbf{2 3 4}) *$ & Mães e avós em coabitação $(\mathbf{n}=\mathbf{1 3 0})$ & Mães e avós sem coabitação $(\mathbf{n}=\mathbf{1 0 4})$ & Valor de $\mathbf{p}$ \\
\hline Intervenção ** & $12,40 \pm 2,51$ & $12,36 \pm 2,38$ & $12,44 \pm 2,64$ & 0,8752 \\
Controle *** & $10,47 \pm 2,26$ & $10,62 \pm 2,39$ & $10,31 \pm 2,13$ & - \\
Valor de $p$ & 0,001 & - & - & -
\end{tabular}

* Amostra total: grupo intervenção versus grupo controle;

** Grupo intervenção: grupo coabitação com avó versus grupo sem coabitação com a avó;

*** Grupo controle: grupo coabitação com avó versus grupo sem coabitação com a avó. 
Tabela 3

Percentual do cumprimento dos Dez Passos para uma Alimentação Saudável: Guia Alimentar para Crianças Menores de Dois Anos nos grupos intervenção e controle. Porto Alegre, Rio Grande do Sul, Brasil, 2006/2009.

\begin{tabular}{|c|c|c|c|c|c|}
\hline & $\begin{array}{c}\text { Cumpriu totalmente } \\
\mathrm{n}(\%)\end{array}$ & $\begin{array}{c}\text { Cumpriu parcialmente } \\
\text { n (\%) }\end{array}$ & $\begin{array}{c}\text { Não cumpriu } \\
\text { n (\%) }\end{array}$ & Total & Valor de $p$ \\
\hline \multicolumn{6}{|l|}{ Passo 1} \\
\hline Intervenção & $14(10,6)$ & $41(31,1)$ * & $77(58,3)$ & 132 & \\
\hline Controle & $8(6,7)$ & $20(16,8)$ & $91(76,5)$ * & 119 & 0,009 \\
\hline \multicolumn{6}{|l|}{ Passo 2} \\
\hline Intervenção & $55(41,7)$ * & $55(41,7)$ & $22(16,6)$ & 132 & \\
\hline Controle & $35(29,4)$ & $42(35,3)$ & $42(35,3)$ * & 119 & 0,003 \\
\hline \multicolumn{6}{|l|}{ Passo 3} \\
\hline Intervenção & $50(37,9)$ * & $75(56,8)$ & $7(5,3)$ & 132 & \\
\hline Controle & $22(18,5)$ & $80(67,2)$ & $17(14,3)$ * & 119 & 0,001 \\
\hline \multicolumn{6}{|l|}{ Passo 4} \\
\hline Intervenção & $40(30,5)$ * & $70(53,5)$ & $21(16,0)$ & 131 & \\
\hline Controle & $17(14,3)$ & $75(63,0)$ & $27(22,7)$ * & 119 & 0,008 \\
\hline \multicolumn{6}{|l|}{ Passo 5} \\
\hline Intervenção & $64(48,5)$ * & $48(36,4)$ & $20(15,1)$ & 132 & \\
\hline Controle & $36(30,3)$ & $48(40,3)$ & $35(29,4)$ * & 119 & 0,004 \\
\hline \multicolumn{6}{|l|}{ Passo 6} \\
\hline Intervenção & $81(62,3)$ * & $44(33,8)$ & $5(3,9)$ & 130 & \\
\hline Controle & $56(47,9)$ & $46(39,3)$ & $15(12,8)$ * & 117 & 0,011 \\
\hline \multicolumn{6}{|l|}{ Passo 7} \\
\hline Intervenção & $74(56,5)$ & $46(35,1)$ & $11(8,4)$ & 131 & \\
\hline Controle & $53(44,5)$ & $49(41,2)$ & $17(14,3)$ & 119 & 0,117 \\
\hline \multicolumn{6}{|l|}{ Passo 8} \\
\hline Intervenção & $13(9,9)$ & $96(73,3)$ & $22(16,8)$ & 131 & \\
\hline Controle & $11(9,2)$ & $76(63,9)$ & $32(26,9)$ & 119 & 0,151 \\
\hline \multicolumn{6}{|l|}{ Passo 9} \\
\hline Intervenção & $78(59,1)$ & $53(40,2)$ & $1(0,7)$ & 132 & \\
\hline Controle & $69(58,0)$ & $50(42,0)$ & $0(0,0)$ & 119 & 0,616 \\
\hline \multicolumn{6}{|l|}{ Passo 10} \\
\hline Intervenção & $54(43,5)$ & $60(48,4)$ & $10(8,1)$ & 124 & \\
\hline Controle & $40(34,2)$ & $63(53,8)$ & $14(12,0)$ & 117 & 0,269 \\
\hline
\end{tabular}

* Associação estatisticamente significativa pelo teste t de Student com cálculo dos resíduos ajustados a 5\% de significância.

ainda amamentavam seus filhos e as que sabiam informar com precisão o momento adequado para a introdução da alimentação complementar tiveram uma probabilidade 4 vezes maior de pertencer ao grupo intervenção 27 . O presente estudo difere do citado anteriormente ao propor uma intervenção realizada por profissionais da área da saúde e envolver diretamente as avós maternas das crianças, além de ter como desfecho a qualidade da alimentação das crianças.

Acredita-se que os escores mais elevados encontrados no cumprimento dos Dez Passos entre as crianças do grupo intervenção se devam a um maior cuidado das mães/avós com a alimentação das crianças e também ao fato de a intervenção ter sido bem-sucedida em aumentar as prevalências de AME e AM no primeiro ano de vida, como relatado em outras publicações 28,29 (vale notar que os dois primeiros passos referem-se especificamente à duração do AME e do AM). Além disso, alguns estudos relatam associação entre maior tempo de AM e AME e melhores hábitos alimentares em pré-escolares 30,31,32, e é possível que essa associação também ocorra em crianças com menos idade.

É importante salientar que a intervenção testada no presente estudo promovia o AME em todos os encontros; por outro lado, apenas no último encontro, quando a criança tinha 4 meses de idade, 
Modelo de regressão de Poisson com estimação robusta para avaliar o efeito da intervenção sobre o cumprimento dos Dez Passos para uma Alimentação Saudável: Guia Alimentar para Crianças Menores de Dois Anos. Porto Alegre, Rio Grande do Sul, Brasil, 2006/2009.

\begin{tabular}{lcc}
\hline Modelo & RR (IC95\%) * & Valor de $\mathbf{p ~}$ \\
\hline 1. Grupo intervenção & $1,93(1,44-2,58)$ & 0,000 \\
2. Modelo 1 + escore de propensão ** & $1,96(1,46-2,60)$ & 0,000 \\
3. Modelo 2 + coabitação com a avó na época da intervenção & $1,95(1,45-2,60)$ & 0,000 \\
\hline
\end{tabular}

IC95\%: intervalo de 95\% de confiança ; RR: risco relativo.

* RR (IC95\%) para escore $\geq$ mediana;

** Variáveis utilizadas para o escore de propensão (todas referentes ao início do estudo): idade, escolaridade, cor da pele e parição maternas; peso ao nascimento e sexo da criança; e coabitação da mãe com o pai da criança.

abordava-se a alimentação complementar saudável com base nas recomendações contidas nos Dez Passos. É possível que o impacto da intervenção tivesse sido maior se ela fosse mantida ao longo do primeiro ano de vida da criança, com mais sessões abordando a alimentação complementar.

A decisão de incluir as avós maternas na intervenção, quando em coabitação, foi baseada no fato de que, segundo alguns estudos, as avós maternas desempenham importante papel na escolha alimentar de seus netos, tanto na decisão quanto ao momento adequado para iniciar a alimentação como participando diretamente da seleção e do preparo dos alimentos 15,18,20,33,34,35,36. Contudo, na amostra deste estudo, a coabitação com as avós maternas, tanto no grupo controle quanto no grupo intervenção, não interferiu no cumprimento dos Dez Passos nos filhos das mães adolescentes. Apesar desse achado, e com base em estudos prévios, acredita-se que as avós maternas, bem como outros familiares tais como os pais das crianças, devam ser contempladas nas estratégias de intervenção que visem à promoção de alimentação saudável em crianças pequenas, em especial os filhos de mães adolescentes.

Apesar de os Dez Passos constarem na Caderneta de Saúde da Criança 37, documento de acompanhamento distribuído a todas as crianças nascidas em território brasileiro, o cumprimento das recomendações na população estudada foi baixo, mesmo para o grupo que sofreu a intervenção. Esse resultado é coerente com dados de inquéritos nacionais sobre o consumo de alimentos em crianças pequenas: baixo consumo de frutas e verduras e alto consumo de alimentos considerados não saudáveis 2,11 . No presente estudo, nenhum passo foi totalmente cumprido por $63 \%$ ou mais das crianças do grupo controle ou intervenção, e apenas os passos 6, 7 e 9, relativos à variedade, consumo diário de frutas, verduras e legumes, e cuidados com a higiene no preparo e manuseio dos alimentos, respectivamente, foram totalmente cumpridos por mais da metade das mães no grupo intervenção. A intervenção aumentou em mais de 10 pontos percentuais o cumprimento de 6 dos 10 passos (passos 2 a 7), relativos à época de introdução, frequência, consistência e variedade dos alimentos complementares, além da flexibilidade de quem alimenta a criança quanto a horários e atitude frente à recusa dos alimentos. Porém, a intervenção não afetou um dos passos mais importantes, a saber, o passo 8, que recomenda evitar açúcar, café, enlatados, frituras, refrigerantes, balas, salgadinhos e outras guloseimas. Levando em consideração que o consumo de refrigerantes, alimentos fritos, doces e salgadinhos por crianças abaixo dos 5 anos é um problema de saúde pública 2,11, a presente intervenção precisaria ser reformulada com o objetivo específico de desencorajar as famílias a oferecer esses alimentos às crianças.

Este estudo tem como mérito seu ineditismo em pelo menos dois aspectos: é o primeiro a avaliar o cumprimento dos Dez Passos para uma Alimentação Saudável: Guia Alimentar para Crianças Menores de Dois Anos e a testar uma intervenção com a participação direta das avós maternas, quando em coabitação, sobre a alimentação de filhos de mães adolescentes. Com relação a esse último aspecto, nosso estudo atende ao apelo feito por Sipsma et al. 14, que, em revisão sistemática para avaliar o efeito de intervenções pró-aleitamento em mães adolescentes de países desenvolvidos, apontaram a necessidade urgente de mais ensaios clínicos testando novas intervenções direcionadas 
especificamente às mães adolescentes, preferencialmente com a inclusão de suas mães e companheirosnas intervenções.

Alguns aspectos metodológicos deste estudo conferem robustez aos resultados, a saber: o delineamento (ensaio clínico randomizado), que reduz a interferência de eventuais fatores de confusão; o seguimento durante o 1o ano de vida, com entrevistas mensais nos primeiros 6 meses de vida das crianças e bimensais no segundo semestre, evitando o viés de memória; e o cegamento dos entrevistadores em relação ao grupo ao qual as crianças pertenciam, prevenindo viés de aferição.

Por outro lado, algumas limitações devem ser levadas em conta. Apesar dos esforços em localizar as famílias, houve um número significativo de perdas no seguimento: em torno de $27 \%$. Tais perdas se deveram, sobretudo, à mobilidade típica da população jovem residente em áreas periféricas de grandes cidades em países em desenvolvimento, típicas de estudos de coorte 38 . Todavia, o delineamento do estudo, o equilíbrio das perdas entre os grupos intervenção e controle, o fato de os participantes que completaram o estudo possuírem características similares, independentemente do grupo, e a inclusão do escore de propensão no modelo estatístico utilizado tornam pouco provável um eventual viés de seleção.

Outro aspecto digno de nota é que no grupo intervenção, em que ocorreram visitas sistemáticas de um profissional de saúde no domicílio dos participantes, hipoteticamente poderiam ter ocorrido mais respostas positivas aos passos porque a entrevistada acreditava que era a resposta esperada ou por medo da perda de algum ganho proporcionado pelas visitas. Esse eventual viés é inerente ao delineamento do estudo, entretanto foi minimizado pelo fato de os entrevistadores não serem as mesmas pessoas envolvidas nas intervenções e pelo fato de que a maior parte dos dados utilizados neste estudo foram coletados a partir do 6 o mês de idade da criança, quando não mais ocorriam visitas domiciliares.

Acredita-se, também, que a possibilidade de contaminação entre os grupos controle e intervenção na maternidade foi pequena, pois eram selecionadas em média 1 ou 2 mães por dia, com pequena chance de virem a trocar informações, além da intervenção ter sido realizada em ambiente privativo.

Finalmente, outro aspecto a ser considerado foi a dificuldade em elaborar um escore que conseguisse traduzir o cumprimento dos Dez Passos preconizados pelo Ministério da Saúde. Essa dificuldade também foi encontrada por outros pesquisadores, quando tentaram criar um escore para avaliar as recomendações alimentares infantis preconizadas pela OMS 39.

É necessário cautela ao generalizar os resultados encontrados neste estudo, pois se trata de um estudo de eficácia, realizado em um único local, envolvendo uma população típica usuária do sistema público de saúde de uma grande cidade brasileira. São necessários estudos em diferentes populações, que testem a efetividade da intervenção em condições que mimetizem a realidade do local. No Brasil, a efetividade da intervenção poderia ser testada pelos agentes comunitários de saúde (ACS) da Estratégia Saúde da Família, cujas atribuições englobam visitas domiciliares regulares. Essa estratégia atualmente cobre mais de 65\% da população brasileira (Portal da Saúde. Histórico de cobertura da saúde da família. http://dab.saude.gov.br/portaldab/historico_cobertura_sf.php, acessado em Dez/2015) e, caso a intervenção testada fosse incorporada às atividades dos ACS, não haveria custos adicionais, muito embora recursos extras seriam úteis à ampliação do número de ACS e aos investimentos em suas qualificações.

Este estudo mostrou que as mães adolescentes são susceptíveis à adoção de práticas alimentares mais saudáveis para seus filhos, desde que orientadas e encorajadas a isso. Apesar de o aconselhamento pró-alimentação saudável ter impactado positivamente na qualidade da alimentação complementar, fazem-se necessários ajustes para que ele consiga modificar práticas comuns, porém danosas, tais como a oferta de alimentos não saudáveis às crianças. Além disso, o estudo confirmou a má qualidade da alimentação das crianças em geral no primeiro ano de vida, salientando a necessidade de investimento em estratégias efetivas para a implementação das diretrizes já preconizadas. 


\section{Colaboradores}

L. M. Nunes foi responsável pela concepção do estudo, coleta, análise e interpretação dos dados, realizou a redação final do artigo, aprovou a versão final a ser publicada e é responsável pela integridade e exatidão de todos os aspectos do trabalho. A. Vigo e L. D. Oliveira contribuíram para a análise e interpretação dos dados, participaram da revisão crítica do conteúdo intelectual, aprovaram a versão final a ser publicada e são responsáveis pela integridade e exatidão de todos os aspectos do trabalho. E. R. J. Giugliani contribuiu para a concepção do estudo, análise e interpretação dos dados, supervisão de todas as etapas do projeto, participou da revisão crítica do conteúdo intelectual, aprovou a versão final a ser publicada e é responsável pela integridade e exatidão de todos os aspectos do trabalho.

\section{Agradecimentos}

Os autores agradecem à Cléa Carvalho, Lilia Refosco e Olga Bica, que participaram da intervenção, às famílias que concordaram em participar do estudo e ao Conselho Nacional de Desenvolvimento Científico e Tecnológico (CNPq), que financiou a pesquisa.

\section{Referências}

1. World Health Organization. Indicators for assessing infant and young child feeding practices. Part 3: country profiles. Geneva: World Health Organization; 2010.

2. Bortolini G, Gubert M, Santos L. Consumo alimentar entre crianças brasileiras com idade de 6 a 59 meses. Cad Saúde Pública 2012; 28:1759-71.

3. Centro Brasileiro de Análise e Planejamento, Ministério da Saúde. Pesquisa Nacional de Demografia e Saúde da Criança e da Mulher PNDS 2006: dimensões do processo reprodutivo e da saúde da criança. Brasília: Ministério da Saúde; 2009. (Série G. Estatística e Informação em Saúde).

4. Bortolini G, Vitolo M, Gubert M, Santos L. Iniquidades sociais influenciam a qualidade e a diversidade da dieta de crianças brasileiras de 6 a 36 meses. Cad Saúde Pública 2015; 31:2413-24.

5. World Health Organization. Obesity and overweight. http://www.who.int/mediacentre/fact sheets/fs311/en/ (acessado em 10/Dez/2015).

6. Singh A, Mulder C, Twisk J, van Mechelen W, Chinapaw M. Tracking of childhood overweight into adulthood: a systematic review of the literature. Obes Rev 2008; 9:474-88.

7. Fall C, Borja J, Osmond C, Richter L, Bhargava S, Martorell R, et al. Infant-feeding patterns and cardiovascular risk factors in young adulthood: data from five cohorts in low- and middle-income countries. Int J Epidemiol 2011; 40:47-62.

8. Ip S, Chung M, Raman G, Chew P, Magula $\mathrm{N}$, DeVine D, et al. Breastfeeding and maternal and infant health outcomes in developed countries. Evid Rep Technol Assess (Full Rep) 2007; (153):1-186.

9. Agostoni C, Decsi T, Fewtrell M, Goulet O, Kolacek S, Koletzko B, et al. Complementary feeding: a commentary by the ESPGHAN Committee on Nutrition. J Pediatric Gastroenterol Nutr 2008; 46:99-110.

10. Horta B, Victora C. Long- term effects of breastfeeding: a systematic review. Geneva: World Health Organization; 2013.

11. Departamento de Ações Programáticas e Estratégicas, Secretaria de Atenção à Saúde, Ministério da Saúde. II Pesquisa de Prevalência de Aleitamento Materno nas Capitais Brasileiras e Distrito Federal. Brasília: Ministério da Saúde; 2009. (Série C. Projetos, Programas e Relatórios).

12. World Health Organization. Global strategy for infant and young child feeding. Geneva: World Health Organization; 2003.

13. Ministério da Saúde. Dez passos para uma alimentação saudável: guia alimentar para crianças menores de dois anos. Um guia para o profissional da saúde na atenção básica. 2a Ed. Brasília: Ministério da Saúde; 2013.

14. Sipsma H, Magriples U, Divney A, Gordon D, Gabzdyl E, Kershaw T. Breastfeeding behavior among adolescent: initiation, duration, and exclusivity. J Adolesc Health 2013; 53:394-400. 
15. Negin J, Coffman J, Vizintin P, Raynes-Greenow $C$. The influence of grandmothers on breastfeeding rates: a systematic review. BMC Pregnancy Childbirth 2016; 16:91.

16. Alder E, Williams F, Anderson A, Forsyth S, Florey C, van der Velde P. What influences the timing of the introduction of solid food to infants? Br J Nutr 2004; 92:527-31.

17. Odom E, Li R, Scanlon K, Perrine C, Grummer-Strawn L. Association of family and health care provider opinion on infant feeding with mothers' breastfeeding decision. J Acad Nutr Diet 2014; 114:1203-7.

18. Santo L, Oliveira L, Giugliani E. Factors associated with low incidence of exclusive breastfeeding for the first 6 months. Birth 2007; 34:212-9.

19. Susin L, Giugliani E, Kummer S. Influence of grandmothers on breastfeeding practices. Rev Saúde Pública 2005; 39:141-7.

20. Aubel J. The role and influence of grandmothers on child nutrition: culturally designated advisors and caregivers. Matern Child Nutr 2012; 8:19-35.

21. Braun M, Giugliani E, Soares M, Giugliani C, Oliveira A, Danelon C. Evaluation of the impact of the baby-friendly hospital initiative on rates of breastfeeding. Am J Public Health 2003; 93:1277-9.

22. Xu Z, Kalbfleisch J. Propensity score matching in randomized clinical trials. Biometrics 2010; 66:813-23.

23. D'Agostino Jr. RB. Propensity score methods for bias reduction in the comparison of a treatment to a non-randomized control group. Stat Med 1998; 17:2265-81.

24. Vitolo M, Bortolini G, Feldens C, Drachler M. Impactos da implementação dos dez passos da alimentação saudável para crianças: ensaio de campo randomizado. Cad Saúde Pública 2005 21:1448-57.

25. Vitolo M, Rauber F, Campagnolo P, Feldens C, Hoffamn D. Maternal dietary counseling in the first year of life is associated with a higher healthy eating index in childhood. J Nutr 2010; 140:2002-7.

26. Scheiwe A, Hardy R, Watt R. Four- year follow-up of a randomized controlled trial of a social support intervention on infant feeding practices. Matern Child Nutr 2010; 6:328-37.

27. Black MM, Siegel EH, Abel Y, Bentley ME. Home and videotape delays early complementary feeding among adolescent mothers. Pediatrics 2001; 107:E67.
28. Dias de Oliveira L, Justo Giugliani ER, Córdova do Espírito Santo L, Meirelles Nunes L. Counselling sessions increased duration of exclusive breastfeeding: a randomized clinical trial with adolescent mothers and grandmothers. Nutr J 2014; 13:73.

29. Bica OC, Giugliani ER. Influence of counseling sessions on the prevalence of breastfeeding in the first year of life: a randomized clinical trial with adolescent mothers and grandmothers. Birth 2014; 41:39-45.

30. Rauber F, Hoffman D, Vitolo M. Diet quality from pre-school to school age in Brazilian children: a 4-year follow-up in a randomised control study. Br J Nutr 2014; 111:499-505.

31. de Lauzon-Guillain B, Jones L, Oliveira A, Moschonis G, Betoko A, Lopes C, et al. The influence of early feeding practices on fruit and vegetable intake among preschool children in 4 European birth cohorts. Am J Clin Nutr 2013; 98:804-12.

32. Burnier D, Dubois L, Girard M. Exclusive breastfeeding duration and later intake of vegetables in preschool children. Eur J Clin Nutr 2011; 65:196-202.

33. Giugliani ER, Espírito Santo LC, de oliveira $\mathrm{LD}$, Aerts D. Intake of water, herbal teas and non-breast milk during the first month of life: associated factors and impact on breastfeeding duration. Early Hum Dev 2008; 84:305-10.

34. Grassley J, Eschiti V. Grandmother breastfeeding support: what do mothers need and want? Birth 2008; 35:329-35.

35. Farrow C. A comparison between the feeding practices of parents and grandparents. Eat Behav 2014; 15:339-42.

36. Bentley M, Gavin L, Black M, Teti L. Infant feeding practices of low-income, AfricanAmerican, adolescent mothers: an ecological, multigenerational perspective. Soc Sci Med 1999; 49:1085-100.

37. Ministério da Saúde. Caderneta de saúde da criança. 10a Ed. Brasília: Ministério da Saúde; 2015.

38. Rothman K, Greenland S. Modern epidemiology. 3rd Ed. Philadelphia: Lippincott Williams \& Wilkins; 2008.

39. Vossenaar M, Doak C, Solomons N. Challenges in the elaboration of a field interview instrument to capture information for the evaluation of adherence to the WHO/PAHO Guiding Principles for Complementary Feeding of the Breastfed Child. Food Nutr Bull 2014; 35: 338-50. 


\section{Abstract}

The objective was to evaluate the effect of a healthy eating intervention targeting adolescent mothers and maternal grandmothers on compliance with dietary recommendations in the first year of life. This was a randomized clinical trial involving 320 adolescent mothers, their infants, and $169 \mathrm{ma}$ ternal grandmothers (when the three generations were living together), randomly assigned to the intervention versus control group. The intervention consisted of six counseling sessions on healthy feeding during the child's first year of life, the first of which held at the maternity ward and the others at 7, 15, 30, 60, and 120 days, at the homes. Information on the infant's diet was obtained monthly during the first 6 months and then every 2 months until the child reached 12 months of age, using a Food Frequency Questionnaire, in addition to questions on mealtimes, consistency of the foods, hygiene, preparation of meals, conservation of foods, and the mother's or grandmother's approach to the child's diet in the presence of illness. The outcome was defined as meeting the Ten Steps to a Healthy Diet: A Food Guide for Children Under Two Years Old, assessed by a score. The mean sum of the scores for each step obtained in the intervention group was higher than in the control group; the intervention doubled the odds of the total score being greater than or equal to the median. Living with grandmother did not influence the score either in the intervention group or the control group. The proposed intervention had a positive effect on meeting the Ten Steps, independently of participation by the maternal grandmother, showing that educational interventions can improve quality of diet for children in the first year of life.

Child Nutrition; Food Habits; Clinical Trial

\section{Resumen}

Evaluar el efecto de intervención pro-alimentación saludable, dirigida a madres adolescentes y abuelas maternas, en el cumplimiento de las recomendaciones alimentarias durante el $1^{\circ}$ año de vida. Ensayo clínico randomizado involucrando a 320 adolescentes, sus hijos y 169 abuelas maternas, cuando en cohabitación, randómicamente se asignan al grupo de intervención o control. La intervención consistió en seis sesiones de asesoramiento en alimentación saludable durante el primer año de vida del niño, la primera en la maternidad y las demás a los 7, 15, 30, 60 y 120 dias, en los domicilios. La información relativa a la alimentación del niño fueron obtenidas mensualmente durante los primeros 6 meses $y$, después, cada 2 meses hasta que el niño completara los 12 meses, mediante un Cuestionario de Frecuencia Alimentaria, además de preguntas sobre horarios de comidas, consistencia de los alimentos, cuidado con la higiene, preparación y conservación de los alimentos y conducta del cuidador con la alimentación del nino en la vigencia de enfermedades. Como desenlace, se consideró el cumplimiento de los Diez Pasos para una Alimentación Saludable: Guía Alimentaria para Niños Menores de Dos Años, evaluado mediante un marcador. La media de la suma de los marcadores de cada paso, obtenidos en el grupo de intervención, fue mayor que la del grupo control; la intervención dobló la oportunidad de que un marcador total fuera mayor o igual a la media. La cohabitación con las abuelas no influenció los marcadores, ni en el grupo de intervención, ni en el grupo de control. La intervención propuesta tuvo un efecto positivo en el cumplimiento de los Diez Pasos, independientemente de la participación de la abuela materna, mostrando que las intervenciones educativas pueden mejorar la calidad de la alimentación de los niños durante el 1 o año de vida.

Nutrición del Niño; Hábitos Alimenticios; Ensayo Clínico
Recebido em 28/Dez/2015

Versão final reapresentada em 18/Jul/2016

Aprovado em 28/Jul/2016 